Copyright $@ 2010$ Institute of Electrical and electronics Engineers, Inc.

All Rights reserved.

Personal use of this material, including one hard copy reproduction, is permitted.

Permission to reprint, republish and/or distribute this material in whole or in part for any other purposes must be obtained from the IEEE.

For information on obtaining permission, send an e-mail message to stds-igr@ieee.org.

By choosing to view this document, you agree to all provisions of the copyright laws protecting it.

Individual documents posted on this site may carry slightly different copyright restrictions.

For specific document information, check the copyright notice at the beginning of each document. 


\title{
Intelligent Encoding of Concepts in Web Document Retrieval
}

\author{
John Zakos ${ }^{1}$, Brijesh Verma ${ }^{1}$, Xue $^{2}{ }^{2}$ and Siddhi Kulkarni ${ }^{3}$ \\ ${ }^{1}$ Griffith University, Australia \\ ${ }^{2}$ University of Queensland, Australia \\ ${ }^{3}$ Nippising University, Canada \\ E-mail: \{j.zakos,b.verma\}@gu.edu.au, siddhik@nipissingu.ca
}

\begin{abstract}
The main aim of the proposed approach presented in this paper is to improve web information retrieval effectiveness by overcoming the problems associated with a typical keyword matching retrieval system, through the use of concepts and an intelligent fusion of confidence values. By exploiting the conceptual hierarchy of the WordNet [1] knowledgebase, we show how to effectively encode the conceptual information in a document using the semantic information implied by the words that appear within it. Rather than treating a word as a string made up of a sequence of characters, we consider a word to represent a concept.
\end{abstract}

\section{Introduction}

Over the years, various information retrieval models and approaches have been proposed. These include Boolean retrieval [2], the vector space model [3], probabilistic models [4], fuzzy set retrieval [5] and neural network models [6]. All these models are unique in their own way and they all have their merits. But the underlying characteristic of all these models is that they are based on a keyword-matching paradigm. There have been attempts to develop concept-based retrieval systems [7-10]. They all attempt to go beyond keyword matching and all of them use a conceptual hierarchy in one way or another. But there has been no comprehensive concept-based model that has been proven to be practical and effective. The main research questions for concept-based information retrieval include: How then can an information retrieval system effectively cater for the nature of language by treating words as concepts? How can a document be represented conceptually? How can these conceptual document representations be searched to perform effective and efficient information retrieval? This paper is organized in 5 sections. The next section describes the proposed approach. Section 3 presents the experimental results. Section 4 presents an analysis and discussion of results and in Section 5 we conclude.

\section{Proposed Approach}

The main idea behind the proposed approach is to treat an occurrence of a term in a document as a concept and to measure the information of that concept and related concepts in the context of the document in appears within. The two main techniques are 1) document encoding, to perform the document indexing, and 2) a neural fusion based search algorithm to perform retrieval.

\subsection{Document Encoding}

The document encoding technique is at the core of the approach and consists of 1) a conceptual encoding technique and 2) a non-conceptual encoding technique. 


\subsubsection{Conceptual encoding}

The conceptual encoding technique generates a conceptual document representation for a document. The goal of the technique is to exploit the conceptual information implied by terms to calculate some concept-based indicators. The two main types of conceptual indicators are 1) concept information content (CIC) and 2) concept importance (CI). 6 mains steps outline the technique: Step 1. Build Conceptual Hierarchy Template, Step 2. Perform Conceptual Marking, Step 3. Calculate Concept Information Content, Step 4. Calculate Concept Importance, Step 5. Combine Conceptual Representations and Step 6. Add to Conceptual Representations to Conceptual Index. When the conceptual encoding technique has completed processing the terms in a document, the resultant conceptual document representation will consist of a set of concepts $C$ where each concept $c \in C$ has a set of associated CIC and CI values.

\section{Conceptual Hierarchy $(\mathrm{CH})$}

To generate the conceptual hierarchy, the WordNet knowledgebase is used. WordNet arranges concepts in a typical is-a style hierarchy, where each parent node in the hierarchy is a conceptual generalization of its child nodes. Since the technique only deals with nouns, we extract only the portion of the WordNet is-a hierarchy that is associated with noun concepts. This extraction procedure produces a conceptual hierarchy that contains a total of 75804 concept nodes (synsets) originating from 9 top-most parent nodes and spreading to a maximum depth of 18 levels. Each node has a local_count (lc) and a subsume_count (sc) variable.

\section{Conceptual Marking (CM)}

$\mathrm{CM}$ marks the $\mathrm{CH}$ with the conceptual information that appears in a document. $\mathrm{CM}$ only uses those terms in a document that can be found in the $\mathrm{CH}$. We call these terms concept words. The technique iterates through every concept word in a particular document. It then marks the $\mathrm{CH}$ with the occurrence of each concept word by either incrementing the local_count or subsume_count at each node in the $\mathrm{CH}$ that is visited. The local_count of concept $\mathrm{c}$ in the $\mathrm{CH}$ represents the amount of information in a document that is directly associated with concept $\mathrm{c}$. The subsume_count of concept $\mathrm{c}$ in the $\mathrm{CH}$ represents the amount of information in a document that subsumes concept $\mathrm{c}$. The input to the technique is the set of terms from one document. The following algorithm describes the technique:

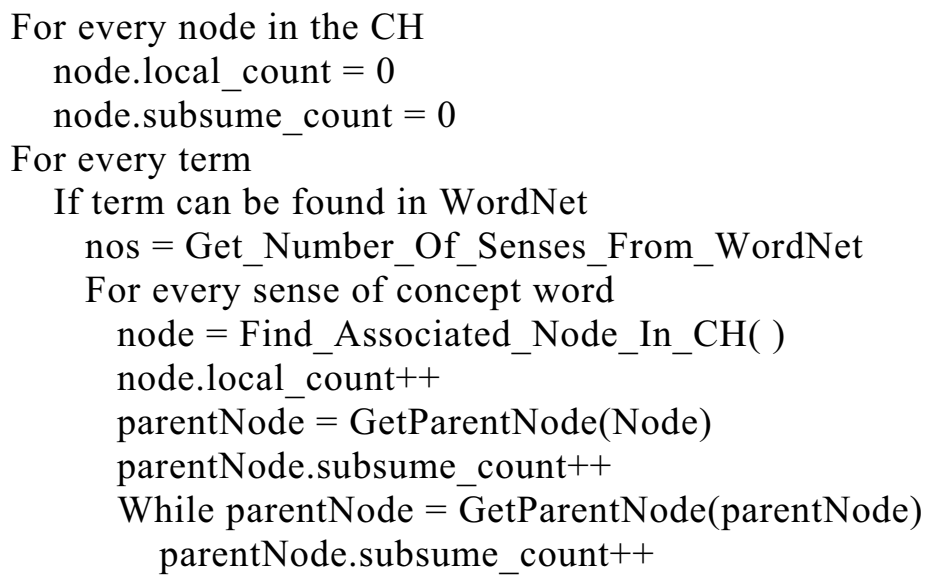




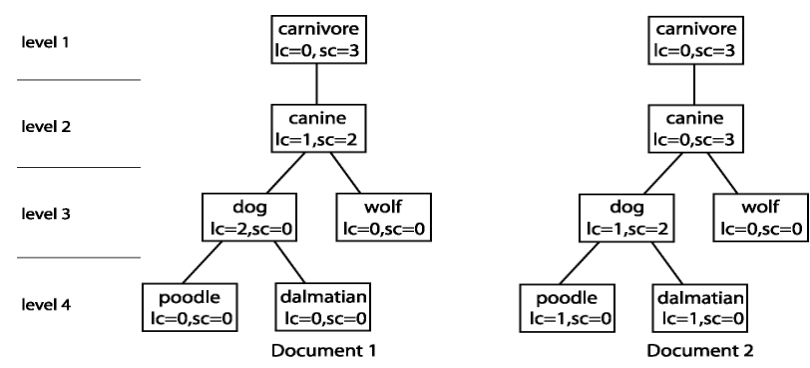

Figure 1. Conceptual hierarchies after conceptual marking

\section{Concept Information Content (CIC)}

Before any of the CIC values can be calculated, it is assumed CM has been performed on the conceptual hierarchy. CIC was developed to give a measure of the amount of information content a particular concept represents in a document. The 2 CIC values that are calculated are CIC_Global and CIC_Local. CIC_Global gives an indication of how much total information content a particular concept represents in a document. The normalisation factor is a constant value that is used for every CIC_Global calculation. The CIC_Global value for concept $\mathrm{c}$ is calculated as follows:

$$
\text { CIC_Global }(c)=\frac{\text { total_count }_{c}}{\text { normalisation_factor }}
$$

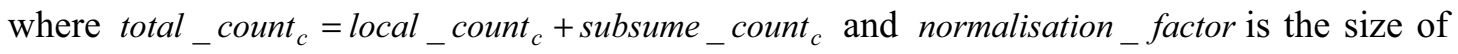
the largest document where size is measured by the number of words.

CIC_Local gives an indication of how much information in a document is directly associated with a particular concept. The CIC_Local value for concept $\mathrm{c}$ is calculated as follows:

$$
\text { CIC_Local }(c)=\frac{\text { local_count }}{\text { total_count }_{c}}
$$

Concept Importance (CI)

CI measures were developed to give a measure of how important a particular concept is in representing the information content of a document. There are two types of CI measures: CI_STDDEV and CI_NOLWE. The intuition behind the technique to calculate CI_STDEV is the more even the spread of information across the levels of the $\mathrm{CH}$ that subsume or supersume a particular concept, then the more important that concept is. We adopt the use of a traditional standard deviation formula and subtract it from 1 to calculate CI_STDEV_Subsume and CI_STDEV_Supersume:

$$
C I I_{-} \operatorname{STDEV}{ }_{-} \operatorname{Subsume}(c)=1-\sqrt{\frac{\sum_{l \in L}(x-\bar{x})^{2}}{n-1}}
$$

where $L$ is the set of levels that subsume $c, x$ is the sum of local_count at level $l$, $\bar{x}$ is the total_count of concept node $c$ divided by the number of levels $n$ in set L. The formula for CI_STDEV_Supersume is the same as CI_STDEV_Subsume except that $L$ is the set of levels that supersume $c$ and $\bar{x}$ is the total_count of top most concept node that supersumes $c$.

The intuition behind the technique to calculate CI_NOLWE is the more levels of local information that subsumes or supersumes concept $\mathrm{c}$ in the $\mathrm{CH}$, the more important concept $\mathrm{c}$ is in representing the document it is associated with. The $2 \mathrm{CI}$ values that are calculated are CI_NOLWE_Subsume and CI_NOLWE_Supersume. CI_NOLWE_Subsume is calculated as follows: 


$$
\text { CI_NOLWE_Subsume }(c)=\frac{\text { evidence_count }}{N O L-1}
$$

where evidence_count is number of levels with local_count values $>0$ that subsume c, and $N O L$ is the number of levels in the in the $\mathrm{CH}$.

CI_DS is the final CI measure that is calculated. It is different from CI_STDEV and CI_NOLWE in that it does not use the conceptual information on the $\mathrm{CH}$ marked by the $\mathrm{CM}$ technique. Instead, it simply uses the depth and sense information of a particular concept to calculate its importance. The less senses a concept has and the shorter the distance of a furthest child node (depth), then the more important that concept is considered to be:

$$
\text { CI_DS(c) }=\frac{1}{\text { NumOfSenses }} * \frac{1}{\text { MaxDepth }}(5)
$$

where

NumOfSenses is the number of WordNet senses for $c$

MaxDepth is the maximum distance to the furthest child node that subsumes $c$ for all senses of $c$. Distance is measured in the number of levels.

\section{Conceptual Representations (CR)}

Once the CIC and CI values have been calculated, the final part of the conceptual encoding technique is to combine the conceptual representations for a given term and add it to the conceptual index. A conceptual representation is the set of conceptual indicators that have been calculated CR $=\{$ CIC_Global, CIC_Local, CI_Subsume, CI_Supersume\}. Since we encode every sense of every term during the CM technique, we now have multiple sets of CR that need to be combined to give one CR for a term. For example, the term dog has 6 difference senses in WordNet and consequently there are 6 different nodes in the $\mathrm{CH}$ corresponding to each of the 6 senses. The challenge at this stage is to combine the six sets of CRs to give us one CR that is a good representative of the concept $\mathrm{dog}$. We perform combination by selecting the CR with the largest CI_Global value. The selected CR is single representation for the term we are combining the CRs for. It is added to the conceptual index.

\subsubsection{Non-Conceptual Encoding}

Non-conceptual encoding generates a standard inverted index using the VSM [11]. This index is only used when keyword matching is incorporated into retrieval. Words that did not appear in a stop list were stemmed using the Porter stemming algorithm [12] and used to generate terms weights using classical tfidf weighting.

\subsection{Neural Fusion Based Search Algorithm}

The search algorithm is responsible for performing retrieval. Its aim is to generate a confidence score for each document that may be relevant to a inputted query. More specifically, it must interpret the CRs in the index to produce a confidence score.

In this research we have investigated conventional fusion based search algorithms and we are currently investigating a neural fusion based algorithm. The search algorithm combines the CIC_Global value and a CI value of a CR to calculate a confidence score for a particular CR. The confidence score using conventional fusion for a $\mathrm{CR}$ is calculated as follows:

$$
C S(C R)=C R . C I C \_ \text {Global } * W(C R)
$$

where weight $W$ is a value generated by combining the CI values of CR. The various weighting schemes that have been developed are listed below: 


$$
\begin{aligned}
& W 1(C R)=C R \cdot C I \_D S, \quad W 2(C R)=A v g\left(\begin{array}{l}
C R \cdot C I+S T D E V I_{-} \text {Subsume }, \\
C R . C I \_S T D E I_{-} \text {Supersume }
\end{array}\right) \\
& W 3(C R)=A v g\left(\begin{array}{l}
C R \cdot C I+N O L W E_{-} \text {Subsume, } \\
C R \cdot C I \_N O L W E_{-} \text {Supersume }
\end{array}\right), W 4(C R)=W 1(C R) * W 2(C R) * W 3(C R), \\
& W 5(C R)=W 1(C R) * W 2(C R), \quad W 6(C R)=W 1(C R) * W 3(C R), \\
& W 7(C R)=W 2(C R) * W 3(C R), \quad W 8(C R)=\operatorname{Avg}(W 1(C R), W 2(C R), W 3(C R)), \\
& W 9(C R)=\operatorname{Avg}(W 1(C R), W 2(C R)), \quad W 10(C R)=\operatorname{Avg}(W 1(C R), W 3(C R)), \\
& W 11(C R)=\operatorname{Avg}(W 2(C R), W 3(C R))
\end{aligned}
$$

The final confidence score for a set CRs for a document is the sum of the confidence scores for each CR:

$$
\text { Document_CS }(S C R)=\sum_{C R \in S C R} C S(C R)
$$

where $S C R$ is the set of CRs for a document.

\section{Experimental Results}

The MED benchmark document collection was used for experimentation. It consists of 1033 documents on medicine and comes with 30 queries, each of which have relevance judgments. We conducted several experiments to test the effectiveness of the approach. The average precision measure was used to measure retrieval effectiveness [13]. The first set of experiments tested the concept-based approach alone with the different weighting schemes.

Table 1. Concept-based experiments with MED

\begin{tabular}{|c|c|}
\hline Weighting Scheme & Average Precision \\
\hline No weighting (baseline) & 0.312 \\
\hline W1 & $\mathbf{0 . 4 4 3}$ \\
\hline W2 & 0.228 \\
\hline W3 & 0.250 \\
\hline W4 & 0.398 \\
\hline W5 & 0.426 \\
\hline W6 & 0.430 \\
\hline W7 & 0.185 \\
\hline W8 & 0.348 \\
\hline W9 10 & 0.387 \\
\hline W11 & 0.393 \\
\hline & 0.235 \\
\hline
\end{tabular}

The second set of experiments combined the concept-based approach with the VSM. The idea here is to combine the concept-based confidence score that a document is assigned for a query with the confidence score (cosine similarity) value calculated by the VSM for the same document and the same query. The results are shown in Table 2.

Table 2. Combining the concept-based approach with the VSM

\begin{tabular}{|c|c|}
\hline Combination & Average Precision \\
\hline VSM & 0.514 \\
\hline VSM + CB & $\mathbf{0 . 5 2 6}$ \\
\hline VSM $^{*}$ CB & 0.524 \\
\hline
\end{tabular}




\section{Discussion of Results}

Looking at Table 1, we can see that the most effective weighting scheme is W1. W2 and W3 actually degrade the retrieval effectiveness by producing precisions of 0.228 and 0.25 that are less than the baseline precision of 0.312 . This is because the MED collection contains short documents with few conceptually related terms. Consequently, many of the CI_STDDEV and CI_NOLWE values would be zero or close to zero. So during retrieval, these values would eliminate the significance of the CIC_Global values. Since W1 is independent of the conceptual information marked on the $\mathrm{CH}$, it is a better indicator of $\mathrm{CI}$ for this collection. Any of the experiments that use W1 as part of the weighting scheme actually improved the precision when compared to the baseline method. These are W4-6 and W8-10 whose precisions were all above 0.312. By using the W1 weighting scheme alone for the concept-based approach and combining the VSM, a significant improvement in the average precision is achieved. While the concept-based approach achieves 0.444 average precision alone when using W1, it achieves 0.526 when combined with the VSM. This is an improvement on the VSM which achieves 0.514 when run alone. The significance of the CIC and CI values will probably be great when the size of the documents in the document collection are larger. This is because there is a greater chance of using words that are conceptually related to each other. The more conceptually related words there are in a document, then more informative the conceptual indicators will be. This should result in improved retrieval performance.

\section{Conclusion}

We have proposed a novel concept-based approach for web information retrieval. We have developed a conceptual encoding technique and proposed several novel conceptual indicators. We have also proposed a search algorithm to interpret these conceptual indicators for retrieval. Experimental experimentation has produced encouraging results that achieve better than the standard VSM.

\section{References}

[1] G. Miller, "WordNet: A Lexical Database," Communications of the ACM, vol. 38, no.11, pp. 39-41, 1995.

[2] R. Baeza-Yates and B. Ribeiro-Neto, "Modern Information Retrieval," Addison Wesley, New York, 1999.

[3] G. Salton, C. Yang and A. Wong, "A Vector Space Model for Automatic Indexing," Communications of the ACM, vol. 18, no. 11, pp. 613-620, 1975.

[4] N. Fuhr, "Probabilistic Models in Information Retrieval," The Computer Journal, vol. 35, no. 3, pp. 243-255, 1992.

[5] D. Lucarella and R. Morara, "FIRST: Fuzzy Information Retrieval SysTem," Journal of Information Science," vol. 17, pp. 81-91, 1991.

[6] T. Doszkocs, J.Reggia and X. Lin, "Connectionist Models and Information Retrieval," Annual Review of Information Science and Technology, vol. 25, pp. 209-260, 1990.

[7] W. Woods, "Conceptual Indexing: a better way to organize knowledge," Technical Report SMLI TR-97-61, Sun Microsystems Laboratories, Mountain View, USA, 1997.

[8] L. Rau, "Knowledge Organisation and Access in a Conceptual Information System," Information Processing and Management, vol. 23, pp. 269-283, 1987.

[9] P. Clark, J. Thompson, H. Holmback and L. Duncan, "Exploiting a Thesaurus-Based Semantic Net for Knowledge-Based Search," in Proceedings of the Twelfth Conference on Innovative Applications of AI (AAAI/IAAI'00), pp. 988-995, 2000.

[10] R. Mihalcea and D. Moldovan, "An Iterative Approach to Word Sense Disambiguation," in Proceedings of FLAIRS-2000, pp. 219-223, 2000.

[11] G. Salton, C. Yang and A. Wong, "A Vector Space Model for Automatic Indexing," Communications of the ACM, vol. 18, no. 11, pp. 613-620, 1975.

[12] M. Porter, “An Algorithm for Suffix Stripping,” Program, vol. 14, pp. 130-137, 1980.

[13] E. Voorhees and D. Harman, Appendix A, in Proceedings of the Seventh Text Retrieval Conference, NIST Special Publication, Gaithersburg, USA, 1999. 